\title{
Percepciones Y Caracterización De Pastizales En Los Cantones Joya De Los Sachas Y Francisco De Orellana
}

\author{
Raúl Lorenzo González Marcillo (Ing. Zootecnista MSc.) \\ Juan Rafael Perez Pupo (Ing. Mecánico PhD) \\ Universidad Escuela Superior Politécnica de Chimborazo \\ Extensión Norte Amazónica de la República del Ecuador
}

Doi: 10.19044/esj.2018.v14n24p311 URL:http://dx.doi.org/10.19044/esj.2018.v14n24p311

\begin{abstract}
This paper focuses on determining the perception and characterization of the pastures in the farms of livestock producers and researchers in the Canton Sachas and Francisco Orellana, Province of Orellana, Ecuador. Their objective was to understand the use and management of Amazonian tropical pastures. Seventeen cattle farms of 8 communities were characterized to observe the characterization of the farmers, land use, use of forage banks, pasture management, and bovine cattle housing. A semi-structured interview was designed to collect primary information about the problem under investigation. When characterizing the farmers in relation to land use, before $47.5 \%$ of the producers were engaged in agriculture, today $85.9 \%$ carry out livestock work with silvopastoral practices. Only $14.1 \%$ were dedicated to agriculture and the production of coffee and cocoa. Regarding the use of feed banks, 35.29\% have more food for their animals, while 5.88\% have better environmental conditions. In addition, $77.6 \%$ of the farmers mentioned that they have improved their production with the use of the feed bank. In conclusion, the results indicate that small and medium producers can benefit from silvopastoral systems as an alternative to improve production and not affect the environment.
\end{abstract}

Keywords: Livestock producers, pasture, fodder, silvopastoral systems, forage bank

\section{Resumen}

El estudio permitió determinar las percepciones y caracterización de los pastizales en fincas de productores ganaderos y de investigadores en el Cantón Sachas y Francisco Orellana, Provincia de Orellana, Ecuador, con el objetivo de conocer el uso y manejo de pastizales tropicales amazónicos. Se 
caracterizaron 17 fincas ganaderas de 8 comunidades, para observar la caracterización de los ganaderos, uso del suelo, uso de bancos forrajeros, manejo de potreros y estabulación de bovinos. Se diseñó una entrevista semiestructurada para recopilar información primaria sobre la problemática investigada. Al caracterizar a los ganaderos, en relación al uso del suelo, antes el $47,5 \%$ de los productores se dedicaban a la agricultura; hoy el $85,9 \%$ realizan labores ganaderas con prácticas silvopastoriles y tan solo el $14.1 \%$ se dedican a la producción de café y cacao. Respecto al uso de los bancos forrajeros, el 35,3\% dispone de mayor alimento para sus animales y el 5,8\% de mejores condiciones ambientales. Además, el 77,6\% de los ganaderos mencionaron que han mejorado su producción con el uso del banco forrajero. En síntesis, los resultados indican que los productores pequeños y medianos pueden beneficiarse de los sistemas silvopastoriles como una alternativa para mejorar la producción y no afectar el medioambiente.

Palabras clave: Productores ganaderos, pastizal, forrajes, sistemas silvopastoriles, banco forrajero

\section{Introduction}

Con el propósito de conocer el rol de los pastizales en fincas ganaderas, se lleva a cabo un trabajo de investigación ecológica en el Cantón Joya de los Sachas y Francisco de Orellana de la Provincia de Orellana Ecuador. Se encuestó y entrevistó a 17 productores y colaboradores, a fin de conocer sus percepciones y caracterización de productores ganaderos, uso del suelo, bancos forrajeros, manejo de potreros y estabulación de ganado. Los productores entrevistados en el Cantón Joya de los Sachas son de las comunidades El Progreso, Freddy Silva, Unión Bolivarense, San Miguel, Pimampiro y Moran Valverde; y, Las Callanas y Guamayacu del Cantón Francisco de Orellana, todos mayores de 49 años de edad y nacidos en diferentes provincias del país; por tanto, sus apreciaciones sobre los pastos y árboles son producto de una larga experiencia.

El alcance de la investigación se basa en conocer la percepción de los productores ganaderos sobre el uso y manejo de los pastizales en los Cantones Joya de los Sachas y Francisco de Orellana, con miras de plantear el uso de paquetes tecnológicos y realizar futuras investigaciones en el uso y manejo de pastizales en sistemas silvopastoriles sostenibles para los ganaderos de esta región de la amazonia ecuatoriana. En la provincia de Orellana, y específicamente en los Cantones Joya de los Sachas y Francisco de Orellana no se han realizado investigaciones de este tipo. Sin embargo, se puede señalar que los suelos de la Provincia de Orellana presentan una baja aptitud agrícola, fragmentación de la tierra, condiciones de pobreza rural, y deficiente 
capacitación a productores en el manejo de sistemas silvopastoriles sostenibles.

La producción ganadera en el trópico practicada por pequeños productores es baja debido a ineficientes prácticas alimenticias y a praderas que se han manejado con sistemas de pastoreo extensivo con poca o nula fertilización y sobrepastoreo, situación que pone en riesgo la sustentabilidad del sistema al provocar degradación de las praderas. Al respecto, en los últimos diez años se ha incrementado la preocupación por el alarmante deterioro de los pastizales en extensas regiones de clima tropical. Entre las causas de esta situación se ha mencionado principalmente la inapropiada regionalización y baja calidad de los pastos cultivados (Franke et al., 2001). Este autor también sostiene que bajo estas condiciones los productores se enfrentan a problemas asociados a la baja calidad y disponibilidad de los pastos utilizados y al inadecuado manejo de los potreros que causa sobrepastoreo, lo que provoca que los animales en la época de sequía y lluvia disminuyan su comportamiento productivo y reproductivo.

La introducción de especies arbóreas en la ganadería, ricas en calidad y adaptadas al clima y suelo, es una alternativa sostenible para intensificar la ganadería y mejorar la producción. Los sistemas silvopastoriles han demostrado la factibilidad de integrar el componente arbóreo en las pasturas como un elemento para mejorar las condiciones productivas de áreas dedicadas a las actividades ganaderas, (Pezo e Ibrahim, 1999) ya que son biológica, económica y ecológicamente más sostenibles que los sistemas tradicionales en el uso de la tierra, sobre todo comparado con los monocultivos de pastizales de gramíneas (Murgueitio et al., 1999). Bajo este contexto, la utilización de especies arbóreas forrajeras tal como la Leucaena leucocephala, establecida como banco proteínico, ha demostrado la factibilidad de integrar el componente arbóreo en la alimentación de los animales ya que apoya la reducción del uso de alimentos concentrados y soporte nutricional. Debido a su mayor producción de biomasa (Jordán et al., 1999) mejora la producción y propicia la intensificación de los procesos de producción ganadera a través de la estabulación o semiestabulación de los animales, disminuyendo de esta manera las áreas ganaderas que puedan ser utilizadas para otros fines agrícolas, forestales o de regeneración natural.

Se plantean alternativas de solución con el uso de la Brachiaria decumbens para suelos rojos y Panicum máximum para suelos negros, que son las gramíneas introducidas de mayor éxito y cobertura en Colombia, Ecuador y en el trópico latinoamericano en general. Pero, a pesar de su gran calidad forrajera, estos materiales han sido persistentemente atacados por una plaga denominada Mión o salivazo (Zulia colombiana, Aneolamia varia), que deteriora considerablemente su productividad y en casos extremos inutiliza totalmente los potreros. Para responder al problema, la investigación con el 
género Brachiaria decumbens y Panicum máximum ha tenido prioridad y apunta hacia el desarrollo de nuevos materiales tolerantes al Mión y con mayor productividad, en términos de carga y producción de carne y de leche, que la Brachiaria decumbens y Panicum máximum tradicional. (Rincón, 2006). En lo que respecta a otra alternativa para los ganaderos que tienen percepciones sobre el uso de árboles forrajeros, se resaltan cinco aspectos; el más importante manifestado fue que podían disponer de mayor alimento para los animales (31.8\%) y el menor que esperaban una mejor condición ambiental (6.7 \%). Al respecto, Simón G. et al. (2005) expresa que la utilización de las especies arbóreas, asociada o en banco de proteína, supera los resultados productivos que se alcanzan cuando se dispone de pastos naturales y/o pastos mejorados no fertilizados, y añade que con estos sistemas se pueden lograr rendimientos de producción. Por otro lado, Aguilar (2008), Calderón (2008), y Jiménez F. et al. (2008) mencionaron que los pastizales diversificados brindan mayor protección al suelo, biodiversidad y servicios ambientales como la mitigación del cambio climático, entre otros.

Estas son las interrogantes que se hacen a los productores ganaderos: ¿Usted remplazaría sus pasturajes por pastos, como por ejemplo las Brachiaria, Saboya, marandu, grama?, ¿Usted renovaría sus potreros con otra variedad de pasto y no con la que actualmente tiene, por qué? Entre las interrogantes hechas a los investigadores técnicos destacan: ¿Qué valoración asignaría a la finca respecto del manejo de pastos?, ¿Conoce de otros estudios en Orellana donde otras instituciones hayan estudiado los pastizales presentes en las fincas?

De acuerdo con lo anterior, la investigación tiene como objetivo general el caracterizar y conocer la percepción de los productores e investigadores sobre el uso de los pastizales en los cantones Joya de los Sachas y Franciscos de Orellana de la Provincia de Orellana Ecuador como una estrategia para intensificar la ganadería.

\section{Materiales y Métodos}

\section{Descripción del área de estudio}

El área de estudio abarcó los territorios de los Cantones Joya de los Sachas y Francisco de Orellana, pertenecientes a la Provincia de Orellana, Ecuador. En el área de estudio existen dos estaciones climáticas predominantes, una tropical húmeda y otra tropical muy húmeda. Posee una estación marcada entre los meses de febrero a agosto, y una precipitación entre 2800 y $3000 \mathrm{~mm}$. La temperatura promedio anual oscila entre $\operatorname{los} 18^{\circ} \mathrm{C}$ y 32 ${ }^{\circ} \mathrm{C}$. La zona de estudio tiene una altitud promedio de $275 \mathrm{msnm}$. Las principales actividades económicas que generan fuentes de trabajo en los municipios son la ganadería y la agricultura, en la que destacan los cultivos de maíz, arroz, café y cacao. 
Fincas ganaderas, percepción, efectos y estrategias del manejo de pastizales

En la zona de estudio se caracterizaron las fincas para conocer las perspectivas del manejo de pastizales, suelo, producción de leche y carne y el medio ambiente, para ello se seleccionaron a productores que se dedican a la ganadería de doble propósito. De los productores seleccionados, mediante un muestreo aleatorio simple (Casas et ál., 2003), se eligieron 17 productores. Se diseñó una entrevista semiestructurada para recopilar información biofísica y socio-económica acerca de las fincas ganaderas.

Percepción de los pequeños productores ganaderos en el manejo de los pastizales

Elaboración del diagnóstico

El diagnóstico se elaboró con 17 preguntas semi-abiertas y estuvo constituido por cuatro rubros: la primera parte indica datos personales de los productores, la segunda se refirió al cambio de uso del suelo (qué realizaban antes, qué realizan ahora, el uso que le dan) la tercera correspondió sobre el establecimiento de bancos forrajeros (el uso del banco forrajeros, el manejo del mismo.) y el cuarto rubro fue acerca del manejo de la estabulación como medida para recuperar potreros.

\section{Aplicación del diagnóstico}

El diagnóstico se aplicó a 17 productores ganaderos de la comunidad el Progreso, Freddy Silva, Unión Bolivarense, San Miguel, Pimampiro, Las Callanas, Guamayacu y Moran Valverde y que cuentan con potreros de entre 10 y 20 años de uso. Cada entrevista tuvo una duración aproximada de entre 15-20 minutos. Para tal efecto, se reunió a los productores en cada finca de la misma comunidad para socializar el diagnóstico y su objetivo, así como conocer quienes contaban con el banco forrajero para la aplicación del diagnóstico.

\section{Sistematización de la información}

La información obtenida en los talleres y entrevistas semiestructuradas fue sistematizada en documentos analíticos y tablas, lo que facilitó el análisis de cada pregunta del diagnóstico.

\section{Análisis de la información}

El análisis de la información colectada no necesariamente requirió de un análisis estadístico convencional, pero si de una forma de análisis alternativa que va más allá de los métodos convencionales representados por la estadística descriptiva. Por esta razón los datos fueron procesados en la hoja de cálculo electrónica del programa Excel. 


\section{Resultados y discusión \\ Características de los productores ganaderos que utilizan sistemas silvopastoriles}

En la Tabla 1 se observa que un $99,93 \%$ de los productores son de sexo masculino, estando el $15 \%$ entre los 41 a 50 años de edad, mientras la mitad de los productores se encuentran entre 31 a 40 años (48\%). Este aspecto es destacable y de vital importancia para la realización de actividades dentro de la finca o implementación de mejoras en los sistemas productivos. Por otra parte, el $78 \%$ de los productores han dedicado entre uno y veinticinco años a trabajar con ganado. Los años de experiencia en la ganadería de los productores son importantes ya que influyen en la adopción de los sistemas silvopastoriles, lo cual facilita que los productores adopten tecnologías agroforestales más complejas (Atta-Krah \& Francis, 1987). La experiencia en el trabajo agropecuario le permite al productor percibir que los diversos recursos se han deteriorado y la tierra no tiene la misma productividad que años atrás, razón por la cual ellos tienden a implementar estrategias de producción nuevas (Villa A et al., 2009). Otro aspecto importante es el nivel de escolaridad de los encuestados. Un menor porcentaje de los productores son analfabetos $(14.3 \%)$, lo que indica que la mayoría saben leer $(85.7 \%)$, facilitando procesos de capacitación tal como se muestra en la Tabla 1, puesto que el $85.7 \%$ tienen conocimientos sobre el manejo de la producción ganadera y sistemas silvopastoriles. Jhonson (1992) menciona que el conocimiento de las nuevas tecnologías principalmente basado en especies arbóreas y arbustivas varían entre los miembros de comunidades entre sí, los productores que han recibido capacitaciones o han accedido a un cierto nivel de educación formal poseen una visión diferente a aquellos que nunca la obtuvieron. Se puede decir que, aunque los productores no presenten, en general, muchos años de educación formal es posible introducir tecnologías mediante capacitaciones frecuentes (Hassan, 1996).

Tabla 1. Características de productores ganaderos que utilizan prácticas silvopastoriles en los Cantones Sacha y Francisco Orellana, Provincia Orellana.

\begin{tabular}{|c|c|c|c|c|c|c|c|c|c|}
\hline \multicolumn{2}{|c|}{$\begin{array}{l}\text { Sexo de productores } \\
\text { encuestados }\end{array}$} & & \multicolumn{2}{|c|}{ Escolaridad } & \multicolumn{2}{|c|}{$\begin{array}{l}\text { Años de experiencia } \\
\text { en ganado }\end{array}$} & \multicolumn{2}{|c|}{$\begin{array}{l}\text { Capacitación en } \\
\text { tecnología }\end{array}$} \\
\hline \multicolumn{2}{|l|}{$\%$} & \multicolumn{2}{|l|}{$\%$} & \multicolumn{2}{|l|}{$\%$} & \multicolumn{2}{|l|}{$\%$} & \multicolumn{2}{|l|}{$\%$} \\
\hline Femenino & 0,07 & 10-20 años & 9 & Profesional & 1 & 1-10 años & 62 & $\mathrm{Si}$ & 85,7 \\
\hline \multirow[t]{3}{*}{ Masculino } & 99,93 & 21-30 años & 28 & $\mathrm{Pt}$ & 11 & 11-20 años & 38 & No & 14,3 \\
\hline & & 31-40 años & 48 & Bachiller & 2 & & & & \\
\hline & & 41-50 años & 15 & & & & & & \\
\hline
\end{tabular}

$\mathrm{Pt}=$ primaria terminada 


\section{Uso del suelo de áreas ganaderas con prácticas silvopastoriles}

En la Tabla 2 se observa que hace 10 años los productores se dedicaban a la agricultura y lo que más producían era café $(47.5 \%)$. Los productores han cambiado el uso de sus tierras y actualmente se dedican más a la ganadería, en razón de que los productores (76\%) poseen más tierras establecidas con gramíneas y especies leñosas las cuales dedican a la ganadería y sólo un 14,1\% son dedicadas a la producción de café y cacao. Esto se debe a que la actividad ganadera tiene actualmente más mercado para sus productos y el costo de los insumos es más bajo; en cambio en la agricultura de hoy en día los insumos son más caros y la producción ya no justifica, producto de suelos infértiles o degradados. Al respecto, Saín (1997) menciona que en el pasado los productores le daban poca importancia a la ganadería; más bien era considerada una actividad complementaria a la agricultura. Sin embargo, esto ha cambiado con el paso del tiempo, pues el productor ha tomado la ganadería como alternativa para mejorar su economía y bienestar social. Lo anterior también coincide con lo estudiado por la FAO (2007), que indica que la ganadería llegó a sustituir en gran parte a la agricultura, esto debido a que el hombre encontró una mejora de vida como consecuencia del aumento de los costos de los insumos para la producción agrícola. Además de eso surgieron muchas plagas y enfermedades que afectan a los cultivos, complican la agricultura y ocasionan la quiebra de muchos productores. De igual manera, el aumento de la población y la demanda de carne hicieron que la ganadería se volviera un mercado más seguro para los agricultores. Los productores realizan cambios en su sistema de producción como respuesta a la necesidad urgente de modernizarse para poder enfrentar la competencia, tanto interna como externa, originada por la apertura de mercados, así como también para que puedan mejorar su calidad de vida en el presente y en el futuro, todo esto en el marco de un escenario de reducidos recursos y servicios gubernamentales tales como créditos limitados, eliminación de medidas proteccionistas, eliminación de subsidios. (Gaitán \& Lacki, 1993).

La mayoría de los productores $(35,29 \%)$ cuentan con una superficie de tierra de 21 a 30 hectáreas en promedio. En esta superficie, la mayoría de ellos $(76 \%)$ tienen sembradas gramíneas y especies arbóreas, éstas últimas en arreglos silvopastoriles. Con relación a las gramíneas, entre las más importantes se encuentran el pasto Dallis (Brachiaria decumbens), Pasto Marandu (Briachiaria brizantha.) y Saboya, (Panicum maximum). Mientras tanto, el $61.3 \%$ tienen especies arbóreas, como Gliricidia sepium. Las gramíneas sembradas en sus potreros obedecen a que ésta es la forma más tradicional de alimentar a los animales. Sin embargo, actualmente se están introduciendo prácticas silvopastoriles (especies arbóreas), lo cual ha causado que los productores recurran a estas prácticas en busca de forraje para sus animales (Pulver et al., 1996). Se aprecia que las especies gramíneas que 
tienen sembradas son introducidas y han sustituido a las pasturas nativas (Grama), porque son bajas en calidad y cantidad y el tener pasturas de baja calidad no beneficia a la producción de los animales; en otras palabras, estos pastos no llenan los requerimientos del animal (CATIE, 1989). Para el caso de la especie arbórea, éstas fueron introducidas para mejorar la alimentación de los animales. Se conoce que estas especies podrían abastecer de mejor forma los requerimientos de los animales, además de que son especies y variedades pratenses y forrajeras de calidad e importantes en la ganadería (Paretas, 1990). En lo que respecta a los arreglos de los árboles forrajeros estos se dividen en tres diferentes tipos: el mayor porcentaje de productores los tienen en banco forrajeros $(80.9 \%)$, mientras que el resto está en pastura en callejones y árboles dispersos en potreros. Esto coincide con Gutteridge y Shelton, (1994) y Pezo e Ibrahim (1998), quienes indican que las formas más estudiadas o el arreglo más común son el banco de proteína, asociación de árboles en toda el área de pastoreo y cercas vivas.

Los productores utilizan las especies arbóreas con diferentes objetivos, el 25,7\% mencionaron que les beneficia para alimento animal, mientras que el $42,6 \%$ encuentran beneficio por su sombra a los animales. Al respecto, Souza et al. (2000), menciona que en Centroamérica un alto porcentaje de finqueros manejan árboles en sus potreros para proveer sombra a sus animales especialmente en las regiones calientes, pues estos ayudan a contrarrestar el estrés calórico de los animales, igualmente Pezo e Ibrahim (1996) señalan que las fincas ganaderas presentan árboles dispersos en potreros para proveer sombra y alimentos para los animales. Dichas plantas mejoran la productividad de los sistemas y suelen favorecer el valor nutritivo del estrato herbáceo y la estructura y fertilidad general de los suelos (Solórzano et al., 1998; Rodríguez et al., 2000). Los sistemas silvopastoriles en diferentes arreglos tienen importancia debido a que proporcionan sombra, alimentos altos en proteína y minerales para los animales, además pueden mejorar el reciclaje de nutrientes, la estructura física y química del suelo, esto incrementa la producción y valor nutritivo de las pasturas, razón por la cual los productores utilizan las especies arbóreas en diferentes arreglos agronómicos (Hernández \& Sánchez, 1998). En esta investigación, el arreglo en la que la mayoría $(68,1 \%)$ de los productores introdujo los árboles forrajeros fue en cercas vivas, debido a que es más fácil el manejo del área establecida, así como también por el corte y ramoneo. Se conoce que los sistemas silvopastoriles más promisorios hasta la fecha son los bancos forrajeros y las asociaciones de árboles en pastizales (Chávez et al., 1996).

La mayoría de los productores prefieren sus especies arbóreas en bancos forrajeros, ya que estos se caracterizan por el cultivo intensivo de una o varias especies de plantas leguminosas que sirven de alimento al ganado. Una característica primordial de estas plantas es su tolerancia a la poda 
frecuente y una buena capacidad de rebrote. Los productores identifican la presencia de árboles y arbustos forrajeros utilizados en sistemas ganaderos y sus diferentes usos y prácticas de manejo (Guillen et al., 2001).

Tabla 2. Uso del suelo de áreas ganaderas con prácticas silvopastoriles en los Cantones

Sacha y Francisco de Orellana, provincia de Orellana.

\begin{tabular}{|c|c|c|c|c|c|c|c|c|c|c|c|c|c|}
\hline \multicolumn{2}{|c|}{ Antes (\%) } & \multicolumn{2}{|c|}{$\begin{array}{c}\text { Ahora } \\
(\%)\end{array}$} & \multicolumn{2}{|c|}{$\begin{array}{c}\text { Superficie } \\
(\%)\end{array}$} & \multicolumn{2}{|c|}{$\begin{array}{c}\text { Especies de } \\
\text { gramíneas } \\
\text { sembradas ( \% ) }\end{array}$} & \multicolumn{2}{|c|}{\begin{tabular}{|c|} 
Especies de \\
arbóreas \\
sembradas (\%) \\
\end{tabular}} & \multicolumn{2}{|c|}{$\begin{array}{c}\text { En qué arreglo } \\
(\%)\end{array}$} & \multicolumn{2}{|c|}{$\begin{array}{l}\text { Principal } \\
\text { objetivo } \\
(\%)\end{array}$} \\
\hline $\begin{array}{l}\text { Vegetación } \\
\text { secundaria }\end{array}$ & 24 & $\begin{array}{l}\text { Vegetación } \\
\text { secundaria }\end{array}$ & 17,7 & De 1 a 10 has. & 23,53 & \begin{tabular}{|l|} 
Pasto Dallis \\
(Brachiaria \\
decumbens) \\
\end{tabular} & 55 & $\begin{array}{l}\text { Mata ratón } \\
\text { Gliricidia } \\
\text { sepium } \\
\end{array}$ & 61,3 & $\begin{array}{l}\text { Pastura en } \\
\text { callejones }\end{array}$ & 5,3 & Suelo & 13,3 \\
\hline Gramíneas & 15 & Gramíneas & 35,1 & De 11 a 20 has & 5,88 & $\begin{array}{l}\text { Pasto Marandu } \\
\text { (Brachiaria } \\
\text { brizantha) }\end{array}$ & 28 & \begin{tabular}{|l|} 
Quiebra \\
barriga \\
Trichantera \\
gigantea
\end{tabular} & 29,4 & $\begin{array}{l}\text { Banco de } \\
\text { forrajero }\end{array}$ & 20,8 & $\begin{array}{l}\text { Alime } \\
\text { nto }\end{array}$ & 25,7 \\
\hline Maíz & 5 & Maíz & 15 & De 21 a 30 has & 35,29 & $\begin{array}{l}\text { Pasto Saboya } \\
\text { (Panicum } \\
\text { máximum) } \\
\end{array}$ & 10 & \begin{tabular}{|l} 
Morera \\
Morus Alba \\
\end{tabular} & 8,1 & $\begin{array}{l}\text { Arboles } \\
\text { dispersos }\end{array}$ & 5,8 & $\begin{array}{l}\text { Somb } \\
\text { ra }\end{array}$ & 18,4 \\
\hline Arroz & 2,5 & Arroz & 10 & De 31 a 40 has & 17,65 & $\begin{array}{l}\text { Pasto miel } \\
\text { (Echynochloa } \\
\text { polystachya) }\end{array}$ & 8 & \begin{tabular}{|l|} 
Leucaena \\
Leucaena \\
leucocepha \\
la
\end{tabular} & 1,2 & $\begin{array}{l}\text { Cerco } \\
\text { vivo en } \\
\text { potreros }\end{array}$ & 68,1 & $\begin{array}{l}\text { mader } \\
\mathrm{a}\end{array}$ & 42,6 \\
\hline Café & $\begin{array}{l}47 \\
5\end{array}$ & Café & 8,5 & De 41 a 50 has. & 5,88 & \begin{tabular}{|l}
$\begin{array}{l}\text { Pasto elefante } \\
\text { (pennisetum } \\
\text { purpureum) }\end{array}$ \\
\end{tabular} & 9 & & & & & & \\
\hline Plátano & 3 & Plátano & 6,1 & De 51 a 60 has & 5,88 & $\begin{array}{l}\text { Pasto mulato } \\
\text { (Brachiaria } \\
\text { Híbrido CIAT } \\
\text { 36087) } \\
\end{array}$ & 3 & & & & & & \\
\hline Cacao & 2 & Cacao & 5,6 & De $61 \mathrm{a}+$ has. & 5,88 & $\begin{array}{l}\text { Pasto Tanzania } \\
\text { (Panicum } \\
\text { máximum) } \\
\end{array}$ & 1 & & & & & & \\
\hline $\begin{array}{l}\text { Sp. } \\
\text { Arbóreas }\end{array}$ & 1 & $\begin{array}{l}\text { Sp. } \\
\text { Arbóreas }\end{array}$ & 2 & & & & & & & & & & \\
\hline
\end{tabular}

Percepción de los productores ganaderos acerca de la tecnología de los bancos forrajeros.

En la Tabla 3 se aprecia las percepciones de los productores con respecto al uso de árboles forrajeros, resaltándose cinco aspectos, de los cuales el más importante fue que podían disponer de mayor alimento para los animales $(35.29 \%)$ y el de menor relevancia fue que esperaban una mejor condición ambiental (5,88\%). Al respecto, Simón et al. (2005) expresa que la utilización de las especies arbóreas, asociadas con gramíneas, supera los resultados productivos que se alcanzan cuando se dispone de pastos naturales y/o pastos mejorados no fertilizados. También manifiesta que con estos sistemas se pueden lograr mayores rendimientos de producción. Por otro lado, Aguilar (2008), Calderón (2008), y Jiménez F. (2008) mencionan que los pastizales diversificados brindan mayor protección al suelo, biodiversidad y servicios ambientales como mitigación del cambio climático, entre otros.

La importancia de utilizar los sistemas silvopastoriles en los hatos ganaderos ha reportado diferentes resultados. Uno de ellos es que se conoce que el uso de leñosas en bancos forrajeros para la suplementación animal ha 
sido significativamente impactante sobre la productividad animal (Jiménez, 2008). Otro resultado importante de la implementación de las especies arbóreas es que estas son capaces de aportar madera, leña, forraje, frutos y postes, de ahí la importancia de los sistemas silvopastoriles, ya que les beneficia también a los productores (Renda, calzadilla, Jiménez, \& Sánchez, 1999).

El productor debe tomar la decisión de integrar y organizar dentro de su finca los múltiples factores en las actividades productivas para adoptar estrategias que beneficien su economía, además de incorporar nueva información referente al potencial de especies leñosas a sus conocimientos empíricos (Prins, 1999). Los sistemas silvopastoriles juegan un papel importante en el incremento de la rentabilidad de las fincas ganaderas (Casasola, 2000; Kaimowitz, 2001; Alonzo e Ibrahim, 2001) al ofrecer beneficios económicos adicionales a la producción bovina, como madera, postes para cercas y suplementos de alta calidad nutricional como forrajes y frutos (Souza de Abreu et al., 2000; Camero et al., 2001; Ibrahim et al., 2001; Navas et al., 2001; Kennedy et al., 2002). Por otra parte, también juegan un papel importante desde el punto de vista de beneficios ecológicos para las fincas y ofrece una alternativa para disminuir la degradación de las pasturas (Szott et al., 2000) y la compactación de suelos al ser comparados con sistemas convencionales (Belsky et al., 1993).

Los productores, de acuerdo a lo que perciben sobre los beneficios que ofrecen los bancos forrajeros, pensarían ampliar la extensión sembrada de árboles forrajeros. Por ello, la mayoría de los productores $(67,71 \%)$ tienen interés en duplicar y ampliar la cantidad que ya tienen, debido a que están satisfechos con el beneficio que han obtenido. Es así como el $100 \%$ de los productores recomiendan a los demás productores ganaderos de la comunidad que aún no cuentan con especies leñosas que los introduzcan a sus terrenos, en vista de que los animales consumen el follaje y fruto, obtienen mayor alimento y menor costo en la alimentación, y también les beneficia en la sombra, leña, postes y mejoramiento de suelos. Lo anterior coincide con Kaimowitz (2001) y Alonzo e Ibrahim (2001), quienes señalan que los sistemas silvopastoriles ofrecen una alternativa para incrementar la productividad de fincas ganaderas al aportar bienes y servicios adicionales a la producción bovina. Los productores, después del tiempo que tienen ya establecidos los sistemas silvopastoriles, ya tienen experiencia y conocimientos sobre árboles en pasturas o en sus potreros, razón por la cual los productores deciden introducir más árboles a sus sistemas de producción (Morrison et al., 1996). Por otra parte, por la experiencia que tienen en ello, la mayoría de los productores $(70,82 \%)$ recomiendan el arreglo de árboles forrajeros en la práctica silvopastoril denominada bancos forrajeros y el resto recomienda en cercas vivas, porque se usan con la finalidad de sostener el 
alambre de púas o liso; además sirven como delimitación de potreros, fuente de madera, alimento para consumo humano y animal (CATIE, 1986). Por otra parte, la importancia de cercos vivos ha sido destacada por varios autores de la región para los diferentes sistemas agroforestales (Montagnini et al., 1992) y en particular para la ganadería (Simón, 1996). La siembra de leñosas perennes como postes para la delimitación de potreros o propiedades (cercas vivas) es una práctica tradicional en América Central (Budowski, 1987; Ivory, 1990). En la Tabla 3 se aprecia las recomendaciones sobre las especies que pueden sembrar los productores que aún no tienen establecidos árboles forrajeros. En este estudio, los productores encuestados recomiendan cuatro especies las cuales son Morera (Morus alba), Mata ratón (Gliricidia sepium), Leucaena (Leucaena leucocephala), Quiebra barriga (Trichantera gigantea), de las cuales Gliricidia sepium es la más recomendada (35,29\%). Esto coincide con Aguilar (2008), Calderón (2008), y Jiménez, F. (2008) quienes mencionan que se debe promover la siembra de especies leñosas forrajeras locales, como Guazuma ulmifolia, Leucaena sp, Gliricidia sepium, Erithryna sp y Brosimum alicastrum, en arreglos agronómicos. Las especies que los productores tienen establecidas en sus fincas son Gliricidia sepium y Leucaena lecucocephala, las cuales son especies arbóreas nativas de la región y por tanto son totalmente adaptadas al clima y al suelo. También son fuente importante de alimento para la ganadería, por el valor nutritivo y forrajero de sus hojas, fruto superior al de las plantas herbáceas (Baumer, 1992; Palma J. et al., 1997).

Tabla 3. Percepción de productores ganaderos acerca de la tecnología de los bancos forrajeros en los Cantones Sacha y Francisco de Orellana Provincia Orellana.

\begin{tabular}{|c|c|c|c|c|c|c|c|c|c|c|c|}
\hline \multicolumn{2}{|c|}{$\begin{array}{c}\text { ¿Qué ventajas } \\
\text { esperaba obtener con } \\
\text { la introducción de } \\
\text { árboles? } \\
(\%)\end{array}$} & \multicolumn{2}{|c|}{$\begin{array}{c}\text { ¿Piensa ampliar } \\
\text { la extensión } \\
\text { sembrada en } \\
\text { banco forrajeros } \\
\text { en los próximos } \\
\text { años? } \\
(\%) \\
\end{array}$} & \multicolumn{2}{|c|}{$\begin{array}{c}\text { ¿Cuánto más? } \\
(\%)\end{array}$} & \multicolumn{2}{|c|}{$\begin{array}{c}\text { ¿Usted } \\
\text { recomienda que } \\
\text { otros productores } \\
\text { usen los bancos } \\
\text { forrajeros? } \\
(\%)\end{array}$} & \multicolumn{2}{|c|}{$\begin{array}{c}\text { ¿Qué arreglo de } \\
\text { árboles (tipo de } \\
\text { sistema } \\
\text { silvopastoriles) } \\
\text { recomendaría usted } \\
\text { que establecieran los } \\
\text { demás productores? } \\
(\%)\end{array}$} & \multicolumn{2}{|c|}{$\begin{array}{c}\text { ¿Qué especie arbórea } \\
\text { recomendaría usted } \\
\text { que otros productores } \\
\text { siembren en la zona? } \\
(\%)\end{array}$} \\
\hline $\begin{array}{c}\text { Mayor } \\
\text { producción de } \\
\text { leche }\end{array}$ & 29,41 & $\mathrm{Si}$ & 64,71 & $\begin{array}{l}\text { La mitad de } \\
\text { lo que hay }\end{array}$ & 41,47 & $\mathrm{Si}$ & 100 & $\begin{array}{l}\text { Banco } \\
\text { forrajero }\end{array}$ & 70,82 & $\begin{array}{c}\text { Morera } \\
\text { Morus alba }\end{array}$ & 23,53 \\
\hline $\begin{array}{c}\text { Aumenta } \\
\text { disponibilidad } \\
\text { de alimento }\end{array}$ & 35,29 & No & 35,29 & El doble & 29,41 & No & 0 & Cercas vivas & 29,18 & $\begin{array}{c}\text { Mata ratón } \\
\text { Gliricidia } \\
\text { sepium }\end{array}$ & 35,29 \\
\hline $\begin{array}{c}\text { Mejor } \\
\text { condición de } \\
\text { animales }\end{array}$ & 17,65 & & & El triple & 17,65 & & & & & $\begin{array}{c}\text { Leucaena } \\
\text { Leucaena } \\
\text { leucocephala }\end{array}$ & 11,76 \\
\hline $\begin{array}{l}\text { Condición } \\
\text { ambiental }\end{array}$ & 5,88 & & & & & & & & & & \\
\hline
\end{tabular}




\section{Manejo de potreros realizado por los productores ganaderos}

La Tabla 4 indica el manejo de potreros por los productores ganaderos. Se observa que los productores cuentan con más de 4 potreros de diferentes tamaños. La mayoría de los productores $(37,5 \%)$ cuentan con 5 a 10 potreros; en menor porcentaje tienen de 11 a 14 potreros $(31,5 \%)$. En lo que respecta al tamaño de potreros, los productores los tienen en un rango de 0,38 a 2 hectáreas $(50 \%)$ y de 3 a 5 hectáreas (50\%). Los productores que tienen de 5 a 10 potreros no pueden brindarles un manejo adecuado a los potreros; es decir, que no le pueden dar los días suficientes de ocupación y de descanso porque necesitan más potreros para realizar la rotación de los animales. Poresta razón , el número de subdivisiones del pastizal es una consecuencia del estudio del tiempo de reposo, de forma tal que permita el manejo del sistema alrededor del punto óptimo de carga (Hernández et al., 1998). En la misma Tabla se observan los días de ocupación que los productores les dan a los potreros. La mayoría de ellos $(52,94 \%)$ los utilizan de 1 a 3 días y el menor porcentaje $(17,65 \%)$ los utilizan de 6 a 8 días hasta que se termina la pastura. Voisin (1963), ha señalado que el tiempo de ocupación deberá ser, preferiblemente, de tres días. Cruz (1996) recomienda menos de 2 días en la época de lluvia y menos de 4 en la época de menor lluvia. Otros autores plantean de 5 a 7 días (Vicente et al., 1974), y hasta 8 días (Senra et al., 1989). De acuerdo a esto, los días de ocupación que los productores les dan a sus potreros es de 1 a 3 días corespondiente al 52,94\% mientras que 17,65\% le dan de 6 a 8 días, por lo que ocurre un sobrepastoreo. Esto provoca pérdida de cobertura vegetal, reducción de la fertilidad del suelo, incremento de la erosión, compactación del suelo y desertificación (Szott et al., 2000). En esta Tabla también se pueden percibir los días de descanso que los productores les brindan a los potreros. El 58,82\% de los productores les dan un mes de descanso y el $29.41 \%$ les dan dos meses. En lo que respecta a los días de descanso, la mayoría de los productores utilizan de uno a dos meses, por tanto, para la época de lluvia, el tiempo de descanso es mayor al recomendado, mientras que para la época de menor precipitación el descanso es insuficiente. Los días recomendados para descanso es entre 18 y 25 días para la época lluviosa y de 35 a 45 días para la época de sequía (Reinoso, 1992). De acuerdo con Senra et al. (1989), no es ventajoso prolongar más de 56 días el tiempo de reposo en los pastos tradicionales en condiciones de secano. También se puede observar la cantidad de animales que tienen los productores encuestados; así se encontró que tienen más vacas $(30,67 \%)$, seguido por terneros $(30.67 \%)$ y el resto se encuentra entre toretes y sementales $(24,62 \%)$. 
Tabla 4. Manejo de potreros por productores ganaderos en los Cantones Sacha y Francisco de Orellana Provincia Orellana

\begin{tabular}{|c|c|c|c|c|c|c|c|c|c|}
\hline \multicolumn{2}{|c|}{$\begin{array}{c}\text { ¿Cuántos potreros tiene y de qué } \\
\text { tamaño es cada potrero en promedio? } \\
(\%)\end{array}$} & \multicolumn{2}{c|}{$\begin{array}{c}\text { iCuántos días ocupa } \\
\text { los potreros? } \\
(\%)\end{array}$} & \multicolumn{2}{c|}{$\begin{array}{c}\text { iCuántos días deja } \\
\text { descansar sus } \\
\text { potreros? } \\
(\%)\end{array}$} & \multicolumn{2}{c|}{$\begin{array}{c}\text { iCuántos animales } \\
\text { tiene? } \\
(\%)\end{array}$} \\
\hline $\mathbf{4 - 5}$ & 31,25 & $0,38-2$ ha & 50 & $1-3$ días & 52,94 & $15-25$ días & 11,76 & Vacas & 30,67 \\
\hline $\mathbf{5 - 1 0}$ & 37,5 & $3-5$ ha & 50 & $4-5$ días & 29,41 & $30-35$ días & 58,82 & Toros & 7,75 \\
\hline $\mathbf{1 1 - 1 4}$ & 31,5 & & & $\begin{array}{c}\text { 6- 8 Acaba la } \\
\text { pastura }\end{array}$ & 17,65 & $37-46$ días & 29,41 & Toretes & 16,87 \\
\hline & & & & & & & & Bacona & 13,8 \\
\hline & & & & & & & & Terneros/as & 30,67 \\
\hline
\end{tabular}

\section{Percepción del manejo de estabulación de los animales por los productores ganaderos}

La Tabla 5 muestra la percepción del manejo de estabulación de los animales por parte los productores ganaderos. Se puede apreciar que el 11,5\% de los productores encuestados señalan que el manejo de estabulación de los animales les ha permitido liberar sus potreros, razón por la cual el $32.2 \%$ de los productores no desean utilizar los potreros que han liberado con la estabulación, mientras que el resto desean establecer más especies arbóreas. Este interés en implementar especies arbóreas se debe a que los productores ya tienen experiencia y conocen el beneficio de los sistemas silvopastoriles para la ganadería. Esto concuerda con Murgueitio et al. (1999), quien indica que los sistemas agroforestales constituyen una parte sustancial de estos procesos de cambio de la ganadería hacia sistemas más amigables con la naturaleza. Por ello, la estrategia es desarrollar Sistemas Silvopastoriles (SSP) para incrementar la productividad de las fincas ganaderas, mientras se liberan terrenos para el establecimiento de bosque secundario o plantaciones forestales.

El manejo de la estabulación es importante porque se liberan los potreros y de esta forma éstos pueden obtener un descanso y recuperación adecuada. De esta manera también pueden ser utilizados para otras actividades importantes, como implementar sistemas agroforestales. Esto coincide con Sánchez M. (1999) quien señala que a través del manejo de la estabulación cambian los esquemas actuales de producción ganadera hacia una intensificación que permita mayor productividad por unidad de área, y de esta manera liberar tierras que puedan ser destinadas a la reforestación u otros fines que resulten en beneficios ambientales. Los productores manifestaron las ventajas de la estabulación de sus animales; así la mayor ventaja mencionada (26.4\%) está relacionada con la recuperación de los potreros degradados que existen en su comunidad evitando con la estabulación que los animales pisoteen los potreros mientras que una de las ventajas menos señalada fue la relacionada a obtener una mayor producción (19,3\%). Por otra parte, el 77.6 $\%$ de los productores manifestaron que han mejorado sus potreros al tener los 
bancos forrajeros. Esto concuerda con Murgueitio e Ibrahim (2001), quienes expresan que los sistemas silvopastoriles donde interrelacionan árboles y arbustos con pastos han probado ser una opción que brinda ventajas cuando se introducen en los sistemas ganaderos en diferentes pisos altitudinales al mejorar las condiciones del suelo, las pasturas, el ganado y el entorno en general. Por tal motivo los productores están interesados en utilizar los potreros liberados y piensan sembrar más árboles forrajeros, pues saben que las arbóreas contribuyen con la economía y la sostenibilidad biótica del sistema de producción (Beer \& Guevara, 2000) y juegan un papel importante en el incremento de la rentabilidad de las fincas ganaderas al ofrecer beneficios económicos adicionales a la producción bovina, como madera y postes para cercas y suplementos de alta calidad nutricional como forrajes y frutos (Casasola, 2000).

Tabla 5. Percepción del manejo de estabulación de animales por productores ganaderos en los cantones Sacha y Francisco de Orellana, Provincia Orellana.

\begin{tabular}{|c|c|c|c|c|c|c|c|}
\hline \multicolumn{2}{|c|}{$\begin{array}{c}\text { ¿El proceso de estabulación } \\
\text { le ha permitido liberar } \\
\text { potreros? } \\
(\%)\end{array}$} & \multicolumn{2}{|c|}{$\begin{array}{c}\text { ¿Qué ventajas le traen al } \\
\text { tener estabulados sus } \\
\text { animales? } \\
(\%)\end{array}$} & \multicolumn{2}{|c|}{$\begin{array}{c}\text { ¿Se han mejorado } \\
\text { sus potreros ahora } \\
\text { que tiene banco } \\
\text { forrajero? } \\
(\%)\end{array}$} & \multicolumn{2}{|c|}{$\begin{array}{c}\text { ¿Ha realizado algo o piensa } \\
\text { realizar algo en los potreros } \\
\text { liberados? } \\
(\%)\end{array}$} \\
\hline $\mathbf{S i}$ & 11,5 & $\begin{array}{c}\text { Consumen mejor el } \\
\text { pasto }\end{array}$ & 17,2 & $\mathrm{Si}$ & 77,6 & $\begin{array}{c}\text { Establecer más } \\
\text { banco forrajeros }\end{array}$ & 19,8 \\
\hline No estabula & 88,5 & Más trabajo & 5,6 & No & 22,4 & $\begin{array}{l}\text { Más árboles } \\
\text { (Mata ratón) }\end{array}$ & 26,4 \\
\hline & & Utiliza poco terreno & 12,5 & & & Sembrar pasto & 25,6 \\
\hline & & Descansar potreros & 16,1 & & & No desea & 32,2 \\
\hline & & Más fácil alimentar & 14,9 & & & & \\
\hline & & Recuperan potreros & 15,3 & & & & \\
\hline & & Mayor producción & 6,9 & & & & \\
\hline & & No estabulan & 11,5 & & & & \\
\hline
\end{tabular}

\section{Conclusión}

En relación a lo antes expuesto, es posible afirmar que los productores de las comunidades el Progreso, Freddy silva, Unión Bolivarense, San Miguel, Pimampiro, Moran Valverde del Cantón Joya de los Sacha y Las Callanas y Guamayacu del Cantón Francisco de Orellana, a través de los programas de extensión y capacitación, han despertado su interés en los sistemas silvopastoriles y cuentan con percepciones claras sobre la utilización de los árboles y el impacto en sus sistemas ganaderos. Igualmente consideran que al implementar y utilizar los sistemas silvopastoriles en los sistemas de producción es una alternativa viable y económica para mejorar los índices de producción animal, principalmente en la época de menor precipitación, que es cuando se presenta la menor productividad animal. Los productores también están conscientes del hecho de que la presencia de los árboles en asociación con gramíneas no sólo aumenta la productividad animal, la disponibilidad y calidad de la gramínea, sino que también hace que exista una mejor 
sostenibilidad del sistema como es el caso de la reforestación. Estos árboles ayudarán a contrarrestar la degradación de sus potreros por medio de la estabulación, ya que con los bancos forrajeros pueden alimentar a los animales en corte y acarreo y de esta manera, en un período de tiempo, se manifestará una mejoría en la producción de sus potreros, en la producción animal y en la calidad de vida de las familias ganaderas.

\section{References:}

1. Aguilar, J. J. (2008). Análisis de los sistemas de producción bovina en la cuenca del río El Tablón, en la zona de amortiguamiento de la reserva de la biosfera La Sepultura, Villaflores, Chiapas. Tesis de Licenciatura. Facultad de Medicina Veterinaria y Zootecnia, Campus III. UNACH. Tutla Gutiérrez, Chiapas, 99 p.

2. Atta-Krah, A. N. \& Francis (1987). The role of on-farm trials in the evaluation composite eriatechnologies: the case of alley farming in Southern Nigeria Agricultural Systems. 23:133.

3. Alonzo, Y.\& Ibrahim, M. (2001). Potential of silvopastoral system for economic dairy production in Cayo, Belize and constraints for their adoption. In: Ibrahim M. ed. Silvopastoral systems for restoration of degraded tropical pasture ecosystems. International Symposium on Silvopastoral System (2001, San José, CR). Memorias. P. 465-470.

4. Belsky, A. J., Mwonga, S. M. et al. (1993). Comparative effects of Isolated Trees on their Undercanopy environments in High-rainfall and Low-rainfall Savannas. Journal of Applied Ecology 30(1): 143-155.

5. Baumer, M. (1992). Trees as browse and to support animal production. In: Andrew Speedy an Pierre-Luc Pugliese (Eds). Legume trees and other fodder trees as protein sources for livestock.

6. Beer, J. \& Guevara, R. (2000). Priority themes in tropical America for agricultural/forestry development: importase of networking XXI IUFRO World congress 200 Sub plenary Sessions. Vol. 1, IUFRO, Kuala Lumpor, Malasia pp. 891-901.

7. Budowsky, G. (1987). Living fences: a widespread agroforestry practice in Central America Pp. 169-178 En: Gholz, H.L (ed) Agroforestry: realities, possibilities and potentials. Njhoff. Dordrecht, The Netherlands.

8. CATIE (1986). Silvicultura de especies promisorias para la producción de leña en América Central: Resultados de cinco años de investigación. Turrialba, Costa Rica. Pp. 171-175.

9. CATIE (1989). Sistemas silvopastoriles para el trópico húmedo bajo. Informe Final primera fase. MAGIDA. CATIE p 56-89. Costa Rica. 
10. Calderón, P. J. (2008). Tendencia de la ganadería bovina y oportunidad para su conversión a sistemas de producción orgánica en el municipio de Tecpatan, Chiapas. Tesis de Licenciatura. Facultad de Medicina.

11. Camero, A. et al. (2001). Improving rumen fermentation and milk production with legume- tree fodder in the tropics. Agroforestry Systems. 51:157-166.

12. Casas, E. et al. (2003). Detection of quantitative trait loci for growth and carcass composition in cattle. Anim Sci. 81:2976-2983.

13. Casasola, F. (2000). Productividad de los sistemas silvopastoriles tradicionales en Moropotentem esteli, Nicaragua. MSc Tesis. CATIE, Turrialba, Costa Rica.

14. Chávez, M. et al. (1996). Efecto del pastoreo restringido en soya perenne sobre la producción de vacas lecheras durante la época de lluvias. Biagro (Venezuela), Revista del decanato de agronomía, UCLA, 8(2): 53.

15. Cruz, C. (1996). Curso de producción de bovinos de doble propósito. Univ. Nacional Autónoma de México pp. 33.

16. FAO (2007). Agroecosistemas son aquellos ecosistemas intervenidos y no intervenidos cuyo uso sea para agricultura, ganadería bosques, y ambientes acuícolas terrestres.

17. Franke, L. A., Valentin, E. et al. (2001). Situacao actual e potencial dos sitemas silvopastoris no estado de Acrel. En: Sistemas agroforestais pecuarios, opciones de sutentabillidae para áreas tropicais EMBRAPA, Brasil P. 19-49.

18. Gaitán, A.J. \& Lacki, P. (1993). La modernización de la agricultura. Los pequeños también pueden. En serie Desarrollo Rural No. 11. FAO.

19. Guillen, J., Jiménez, F. et al. (2001). Ganadería indígena en el norte de Chiapas.

20. Guttridge, R. C. \& Shelton, H. M. (1994). El Campo y el potencial de las leguminosas arbóreas en la Agroforesteria en desarrollo sostenible. Universidad Autónoma Chapingo. Chapingo, México. Pp. 17-43.

21. Hernandez, M. \& Sánchez, S. (1998). Aporte del follaje arbóreo en la producción de guinea y en la fertilidad del suelo. III taller Internacional silvopastoril. Los árboles y arbustos en la ganadería, Matanzas, CU. 130-132 p.

22. Hernández, D. et al. (1998). Explotación de un sistema silvopastoríl multiasociado para la producción de leche. "Los árboles y arbustos en la ganadería", Matanzas, Cuba, p.214.

23. Hassan, R. M. (1996). Planting strategies of maize farmers in Kenya: a simultaneous equations analysis in the presence of discrete dependent variables. Agricultural Economics 15: 137-149. 
24. Ibrahim, M., Beer, J. et al. (2001). Sistemas silvopastoriles para la restauración de ecosistemas de Pasturas tropicales Degradados. In: Simposio Internacional sobre sistemas Silvopastoriles y segundo Congreso sobre Agroforesteria y producción ganadera en América Latina Turrialba - Costa Rica.

25. Ivory, D. A. (1990). Major characteristics, agronomic features and nutritional value of shrubs and tree fodders. In: C Devendra (ed ). Shrubs and Tree Fodders for Farm Animals, Proceedings of a Workshop held in Denpasar, Indonesia, July 24-29, 1989. IDRC, Ottawa, Canada. p. 22-38.

26. Jiménez, F. et al. (2008). Ganadería y conocimiento local de árboles y arbustos

27. forrajeros de la selva Lacandora, Chiapas, México. Zootecnia Trop. 26(3). 333-337.

28. Johnson, M. (1992). Reconocimiento el valor del conocimiento tradicional. CIID.

29. Jordán, H., Mejía, R. et al. (1999). Tecnología para la utilización de Leucaena leucocephala como banco de proteína en la producción de leche y hembras en desarrollo en el trópico.

30. Kaimowitz, D. (2001). Will livestock intensification help save Latin America's Tropical Forest? In: Angelsen; Kaimowitz, D. eds. Agricultural Technologies and Tropical Deforestation. Wallingford, UK, CABI. p 1-20.

31. Kennedy, P. et al. (2002). Utilization of tropical dry season grass by ruminants is increased by feeding fallen leaf of siris (Albizia lebbeck). Animal Feed Science and Technology. 96:175-192.

32. Murgueitio, E., Rosales, M. et al. (1999). Agroforesteria para la producción animal sostenible. Centro para la investigación en sistemas sostenibles de producción Agropecuaria CIPAV. Cali, Colombia 67 pp.

33. Morrison, B. et al. (1996). Incorporating indigenous knowledge of fodder trees into small- scale silvopastoral systems in Jamaica.Agroforestry Systems 34:101- 117.

34. Montagnini, F. et al. (1992). Sistemas agroforestales, principios y aplicaciones en los trópicos. Organización de Estudios Tropicales, Costa Rica, 622 p.

35. Murgueitio Enrique e Ibrahim Muhammad (2001). Agroforestería pecuaria para la reconversión de la ganadería en Latinoamérica. LIVESTOCK RESEARCH FOR RURAL DEVELOPMENT, vol 13.2, 2001.

36. Oquendo, G. (2002). Tecnologías para el fomento y explotación de pasto y forrajes. Ed. Agro Acción Alemana. Ministerio federal para la 
cooperación económica y el desarrollo y asociación cubana de producción animal. La Habana- Cuba. 112 pp.

37. Paretas, J. (1990). Ecosistemas y regionalización de pastos en cuba, ministerio de la Agricultura, Cuba. 178 pp.,1990.

38. Palma, J. et al. (1997). Aproximación al estudio de la vegetación arbórea del estado de Colima. Décimo Aniversario de Avances de Investigación. Trópico 97. Barra de Navidad, Jalisco. pp 89-91.

39. Pezo, D. \& Ibrahim, M. (1996). Sistemas silvopastoriles: Una opción para el uso sostenible de la tierra en sistemas ganaderos In: 1er. Foro internacional sobre pastoreo en zonas tropicales. Veracruz. México. 79. Noviembre FIRA Banco de México, Morelia. México 29 p.

40. Pezo, D. \& Ibrahim, M. (1998). Sistemas silvopastoriles. Colección de módulos agroforestales, No. 2 CATIE p. 15.

41. Pezo, D. \& Ibrahim, M. (1999). Sistemas silvopastoriles módulo de enseñanza agroforestal $\mathrm{N}^{\circ}$ 2. 2 ed. Turrialba. C.R. Proyecto agroforestal CATIE/GTZ.

42. Pulver, E., Araya, L. et al. (1996). Conservation-effective Livestock production. Narmap, Belize. 7 p.

43. Prins, K. (1999). Rutas y redes de extensión Agroforesteria de las Américas 6(21) 21-25.

44. Rincon, A. (2006). Factores de degradacion y tecnologia de recuperacion de praderas en los llanos orientales. CORPOICA, primera edicion, Colombia.

45. Rodríguez, I. et al. (2000). Comportamiento de la macrofauna del suelo en un sistema de ceba de toros con utilización de la Leucaena. En: IV Taller Internacional "Los árboles y arbusto en la ganadería tropical". Estación Experimental de Pastos y Forrajes Indio Hatuey. Cuba. p. 356.

46. Reinoso, M. (1992). Estudio sobre la producción de leche y pasto con PRV en la estación seca. Tesis en opción al grado de Especialista en Nutrición y Manejo de Rumiantes, ICA-ISCAH, La Habana. Cuba, p. 70.

47. Renda, A., Calzadilla, E. et al. (1999). El silvopastoreo en Cuba Agroforesteria para la producción animal en América Latina. Memorias de una conferencia electrónica. Estudio FAO Producción y Sanidad Animal, Roma pp 369-389.

48. Sánchez, M. (1999). Sistema agroforestales para intensificar de manera sostenible la producción animal en Latinoamérica tropical. En:. Agroforestería para la producción animal en Latinoamérica. Estudio FAO sobre producción y sanidad animal 143. (Ed: M Sánchez y M Rosales) Roma pp 1-36. 
49. Saín, G. (1997). Seminario taller. La adopción de tecnología: la perspectiva del agricultor y sus implicaciones para la elaboración de políticas. San José CR. 350 p.

50. Simón, L. (1996). Utilización de árboles leguminosas en cercas vivas y en pastoreo. En sistemas silvopastoriles, alternativa para una ganadería moderna y competitiva. Memorias Segundo seminario Internacional, Ministerio de Agricultura- CONIF. Santafé de Bogotá. Colombia pp 31-42.

51. Simón, L., Lamela, L. et al. (2005). Aspecto relevante de la producción animal bovina en sistemas silvopastoriles de bajos insumos. Producción Animal Tropical I Congreso Internacional de Producción Animal, III Congreso Internacional sobre mejoramiento Animal, I congreso Internacional sobre ganadería Sostenible, La Habana - Cuba (ISBN 959-7164-67-1).

52. Senra, A., Ugarte, J. et al. (1989). Determination of the number of paddocks necessary for the rotation of grazing cows XVI Int. Grassed. Congr. Nice, France p 1136.

53. Szott, L. \& Ibrahim, M. (2000). The hamburger connection hangover cattle pasture land degradation and alternative land use in Central América (Serie Técnica. Informe técnico/CATIE $n^{\circ} 313$ ). Turrialba, CATIE-DANIDA-GTZ, 71PP.

54. Solórzano, N. \& Arrends, E. (1998). Composición química del pasto estrella en sombra de samán, Rev. Unellez de Ciencia y Tecnología 16: 1.

55. Souza de Abreu, A. M., Ibrahim, M. et al. (2000). Caracterización del componente arbóreo en los sistemas ganaderos de la fortuna de San Carlos. Costa Rica. Agroforesteria en las Américas 7(26): 53-56.

56. Souza de Abreu et al., 2000; Camero et al., 2001; Ibrahim et al., 2001a; Navas et al., 2001; Kennedy et al., 2002.

57. Villa, A. et al. (2009). Utilización de guácimo (guazuma ulmifolia Lam.) como fuente de forraje en la ganadería bovina extensiva del trópico mexicano. Tropical and 91 subtropical Agroecosystems, vol. 10, num. 2. Mayo- agosto, pp. 253261.

58. Voisin, A. (1963). Productividad de la hierba. Edit. Tecnos. S.A. Madrid, España.

59. Vicente, C. et al. (1974). Intensive grassland management in the humid tropics of Puerto Rico. Agric. Exp. Univ. Puerto Rico. Bull. 233, p.69. 